\title{
“ Creciendo de corazón. Una propuesta educativa para el desarrollo de los talentos múltiples"
}

\section{"Growing from the heart. An educational proposal for the development of multiple talents"}

\author{
DOI: $10.46932 / \mathrm{sfjdv} 2 \mathrm{n} 4-022$
}

Received in: March 1st, 2021

Accepted in: May 30th, 2021

\section{Esperanza Meseguer Navarro}

Master de Neurodidáctica por la Universidad de Barcelona Diplomado en óptico y Optometría por la Universidad de Alicante

Maestra por la especialidad de Infantil y adaptación al grado por la universidad de Almería y la Universidad Europea de Valencia

Funcionaria de Carrera de la Región de Murcia.LCE Holanda, representante de la Lengua y Cultura

Española en Holanda para el Ministerio de Educación y Formación Profesional en el Exterior Adress. C/Ramón Gallud 16 2D 03181 Torrevieja Alicante Spain

E-mail:: emeseguer@yahoo.es

\section{QUÉ ES CRECIENDO DE CORAZÓN}

Creciendo de Corazón, es una propuesta para desarrollar los talentos múltiples desde una perspectiva neurodidáctica enfocada a la educación infantil. Creciendo de corazón fundamenta su programa con un tipo de aprendizaje basado en proyectos que parte de a motivación del alumno/a para desarrollar los talentos múltiples desde el juego con un diseño universal de aprendizaje accesible a todo tipo de formas de aprendizaje y con material adaptado a la COVID, que permite ser desinfectado fácilmente y descargado de una plataforma para ser impreso, es decir, resulta ser una propuesta de aprendizaje híbrida.

En Creciendo de corazón la finalidad de la propuesta cobra siempre un sentido social, enfocando el producto final en una actividad de aprendizaje-servicio que permita contribuir a la sociedad.

Todos los talentos se entrenan por igual buscando su desarrollo y permitiendo que todos encuentren sus fortalezas.

Este programa de propuestas prácticas tiene un origen neurodidáctico en el que la motivación, las emoción con valencia positiva, la sorpresa y la tecnología como herramienta de aprendizaje juegan un papel fundamental, enfocado a desarrollar los objetivos de desarrollo sostenible.

El movimiento, el apoyo visual y la distribución e itinerarios de aprendizaje son ejes fundamentales de esta propuesta que tiene como base una escuela con cerebro (Guillén 2017).

El entrenamiento de las competencias emocionales es otra cualidad que gira entorno a un 
aprendizaje equilibrado, activo, participativo y cooperativo que hace visible el pensamiento mediante habilidades y rutinas de pensamiento (Perkins, 2008.).

En definitiva es una propuesta que busca desarrollar una mentalidad de crecimiento frente a una mentalidad fija (Dweck, 2012).

\section{UNA PROPUESTA PRÁCTICA CREATIVA Y REAL. "LOS SECRETOS QUE SE ESCONDEN EN TU CORAZÓN"}

\subsection{UNA INICIATIVA QUE RESPONDE A UNA NECESIDAD DE AYUDA A LA POBLACIÓN DE YIBUTI QUE PRECISA DE INSTRUMENTAL PARA CURAS}

El proyecto surge del interés y el miedo que suscita la sangre cuando los niños y niñas se hacen alguna herida en el cole. La sangre resulta muy llamativa para todos los niños y niñas que acuden a observarla de cerca cuando le sale a algún niño al hacerse una herida.

Ésta siempre viene asociada al miedo y al dolor por lo que decidimos investigar un poco más sobre ella y sobre nuestro corazón que es el símbolo de nuestra clase pero que realmente no conocíamos su verdadera forma o el porqué de su color.

Agradecer a los sanitarios el trabajo realizado durante la pandemia exponiendo sus vidas, es una de las razones de este proyecto que nos traslada al mundo de la medicina, la investigación, la biotecnología y la solidaridad con los más necesitados.

"La Estela de Ayo" es un a Asociación sin ánimo de lucro a la que nos encontramos muy vinculados ya que se creó tras la muerte de Ayo, un papá piloto de la base del Ejército del Aire donde trabajan la mayoría de familias de nuestros alumnos/as. Ayo realizaba campañas de recogida de recursos para los niños y niñas de Yibuti donde volaba con frecuencia para realizar una misión. En uno de sus viajes el avión se estrelló. Hoy seguimos su estela realizando la labor que él comenzó y ahora son otros compañeros del piloto los que transportan la mercancía que en lugares como en nuestro cole recogemos. La Estela de Ayo, nos facilita con frecuencia material gráfico de la escuela de Yibuti, de los niños/as, de sus casas, cuando llega la mercancía...a través de sus ojos, podemos ver lo que sucede en Yibuti y cómo reciben lo que recolectamos en clase.

La necesidad en esta ocasión vine por la entrada al país de unos treinta niños que cruzaron la frontera de Etiopía con heridas sangrantes y que por la falta de material de curas, se infectan y degeneran enfermedades importantes. Por esta razón, nos vemos con la necesidad de saber cómo cicatrizan las heridas y recoger material que pueda ayudarles a curar y cicatrizar esas heridas

El proyecto da inicio con una actividad de sorprendizaje en la que un papá enfermero nos trae al cole un corazón de vaca y nos explica todas las partes de las que está compuesto, las válvulas, ventrículas, 
aurículas, venas arterias,... y cómo funciona el corazón. De esta modo tan gráfico y significativo comprendimos cómo era capaz de distribuir toda la sangre hasta todos los rincones de nuestro organismo y del porqué del latido de nuestro corazón. Nos sorprendió mucho que ¡ni durmiendo el corazón se paraba!.

Aprendimos cómo es de grande nuestro corazón y que el animal del que fuera ese corazón debía ser muy grande ya que era mucho más grande que nuestro puño. También conocimos los diferentes grupos sanguíneos. Los niños aprendieron con sus familias su grupo sanguíneo y en clase coloreamos un medallón con el grupo sanguíneo de cada uno.

Este medallón fue fundamental para realizar el juego de las compatibilidades en el que compartimos bocadillo con los grupos sanguíneos compatibles al nuestro.

Solo un niño de nuestra clase resultó ser donante universal y también aparecieron grupos sanguíneos más comunes y los menos frecuentes.

Cada niño aprendió su grupo sanguíneo de forma significativa y a quién podía donar sangre en situaciones que fuera necesario.

El enfermero nos explicó la importancia de donar sangre cuando sean mayores y de animar a sus padres y familiares para donar sangre ya que en estos momentos de crisis que estamos viviendo está siendo muy valiosa.

Otra actividad sorprendente que cautivó nuestra motivación por descubrir más sobre la sangre fue compararla con el zumo de remolacha. Desarrollar habilidades de pensamiento a través de organizadores gráficos es fundamental para desarrollar el pensamiento y hacerlo visible. Esto, nos ayudó a conocer mejor algunas características de la sangre como las plaquetas, los glóbulos blancos, los glóbulos rojos y el plasma.

Conocimos el funcionamiento de cada uno de ellos y cómo con la limpieza, la desinfección y porqué tapar algunas heridas ayuda a su recuperación y que las plaquetas actúen.

Tras las actividades iniciales que despertaron nuestra motivación y entusiasmo, comenzaron a desarrollar sus talentos con los itinerarios de aprendizaje que aparecieron dispuestos en sus bandejas correspondientes.

Cada alumno/a se acerca a las bandejas de su equipo para realizar los itinerarios en el orden deseado con la condición de realizarlos todos.

Los talentos trabajados fueron: lingüístico, lógico-matemático, naturalista, emocional, musical, visual-espacial, cinestésico y digital 


\section{OBJETIVOS:}

- Aprender con sentido y significado aportando los conocimientos a la sociedad para ayudar a los desfavorecidos

- Entrenar y desarrollar los diferentes talentos partiendo de una temática motivadora y que despertó gran interés en los niños/as

- Saber el propio grupo sanguíneo para poder decirlo en caso de emergencia y saber qué grupos sanguíneos son aceptados por cada uno

- Conocer las compatibilidades de los grupos sanguíneos y algunas funciones de los componentes de la sangre: glóbulos rojos, blancos, plaquetas, plasma

- Desarrollar la inteligencia de manera integral desarrollando todos los talentos

- Aprender de forma significativa con un corazón de verdad pudiendo tocar lo que tenemos por dentro

- Aprender desde el asombro el interés y la ayuda a los demás, creando una esfera de compromiso con la sociedad y con el planeta así como de agradecimiento a los sanitarios que tanto nos han cuidado en la pandemia

- Utilizar los conocimientos aprendidos para aportar a la sociedad, a los más desfavorecidos y generar un cambio en las personas que les rodean

\section{Producto final:}

- Grabamos un vídeo de agradecimiento a las enfermeras, mujeres de verde y enfermeros, sanitarios, militares...que grabamos durante la cuarentena que le hacemos llegar a los hospitales LA EVALUACIÓN...

Durante el proceso el alumnado dispone en cada espacio de aprendizaje de un registro de autoevaluación y valoración de la propuesta de aprendizaje y valora su trabajo en función de su implicación utilizando un emoji.

En cada propuesta de aprendizaje el alumno/a sabe cuál es el concepto que ha de adquirir y dispone de un panel de entrenamiento para reforzarlo si lo precisa. Por otro lado, el docente realiza un registro rubricado del grado de adquisición de los aprendizaje, disponiendo de la etiqueta de destacado permitiendo hacer visible cómo todos los alumnos/as son capaces de desatacar en un talento. Además a los padres les envío un google form para valorar la experiencia y la metodología de aprendizaje de sus hijos/as así como su grado de satisfacción 


\section{BIBLIOGRAFÍA}

- $\quad$ Dweck C. (2012). “Mindset. La actitud del éxito”. Sirio

Elizondo C. (2020). "Hacia la inclusión educativa en la universidad:Diseño universal para el aprendizaje y la educación de calidad”. Octaedro

- $\quad$ Perkins D (2008). “La escuela inteligente”. Gedisa

Gardner, H. (2006). Schaler, Jeffrey A., ed. "A Blessing of Influences" in Howard Gardner Under Fire. Illinois: Open Court.

Gardner, H. (1989). To Open Minds: Chinese Clues to the Dilemma of American Education. Nueva York: Basic Books.

Guillén, J. (2017). "Neuroeducación en el aula de la teoría a la práctica”. Cegal

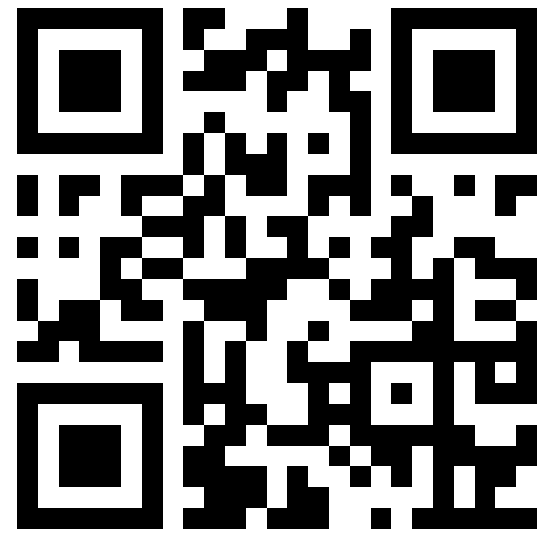

